\title{
Empirical Evaluation of Overlap Requirements of Adjacent Radio Cells for Zero Delay Handover
}

\author{
Marc Emmelmann and Hyung-Taek Lim \\ Technical University Berlin \\ Telecommunication Networks Group TKN \\ Berlin, Germany \\ Email: emmelmann@ieee.org, lim@tkn.tu-berlin.de
}

\begin{abstract}
Velocity has a non-neglectable influence on the handover delay experienced by a user if the handover is triggered on a radio-signal-measurement (RSM) based handover scheme. Previous publications have theoretically derived the minimal required overlap of adjacent radio cells for a zero delay handover if signal averaging (low-pass filtering) and a hysteresis margin are employed in the handover decision process. This paper presents for the first time a practical verification of those results by employing real world channel traces from a high-speed train scenario and considering the effects of an existing radio front-end due to the latter's receiver sensitivity. Also, in addition to related work, we adapt a time-discrete analytical model for comparing theoretical findings with channel trace based results which is closer to an implementation sampling the radio channel's quality at distinct time intervals.
\end{abstract}

\section{INTRODUCTION}

Next generation wireless local area networks (WLANs) provide throughput rates of several $100 \mathrm{Mbit} / \mathrm{s}$ by improving the efficiency of the MAC and incorporating advanced MIMO system. Even though the IEEE standardization body is still working on the final ratification of 802.11n [1] incorporating these features, the IEEE 802.11 working group in conjunction with other research projects have already started working on further enhancements providing even higher throughput [2]-[4]. Apart from exploring multi-user diversity including dynamic OFDM schemes [5]-[8], devices will also operate within the $60 \mathrm{GHz}$ frequency band to achieve very high throughput. As emitted radiation power is limited, the coverage area of each WLAN radio cell will shrink especially for the higher frequency band.

Along with the fact of extremely reduced cell sizes comes another challenge: handover is not longer a seldom event. As attenuation limits the cell size of an office environment to only a few meters, the dwell time of a mobile user in a cell may be in the order of only a few seconds emphasizing the need for an efficient scheme providing a seamless handover. Accordingly, researchers intensively focused on approaches reducing the time spent on the handover. Tabl I [9] classifies the best, currently published approaches according to the different handover phases.

Accordingly, the delay associated with handover detection and decision may be reduced to $0 \mathrm{~ms}$. The underlying system model assumes that the decision when to switch from one AP to the other is based on a radio-signal-strength-based
TABLE I

Achievable Delay Per Handover Phase - State of the Art

\begin{tabular}{|c|c|c|c|c|}
\hline Ref. & $\begin{array}{c}\text { Detec- } \\
\text { tion }\end{array}$ & $\begin{array}{c}\text { Network } \\
\text { Discovery }\end{array}$ & $\begin{array}{c}\text { Deci- } \\
\text { sion }\end{array}$ & $\begin{array}{c}\text { Link Re- } \\
\text { Establishment }\end{array}$ \\
\hline $\begin{array}{c}{[10],[11]} \\
{[12],[13]} \\
{[14]}\end{array}$ & $0 \mathrm{~ms}$ & $\mathrm{n} / \mathrm{a}$ & $0 \mathrm{~ms}$ & $\mathrm{n} / \mathrm{a}$ \\
\hline$[15],[16]$ & \multicolumn{3}{|c|}{$3.5 \mathrm{~ms}$ (plus $10 \mathrm{~ms}$ for channel switch) } \\
\hline$[17]$ & \multicolumn{3}{|c|}{$\begin{array}{c}\ll 10 \mathrm{~ms} \\
\text { (only single frequency } \\
\text { networks; low user mobility) }\end{array}$} & $\mathrm{n} / \mathrm{a}$ \\
\hline
\end{tabular}

(RSM-based) algorithm. The latter employs a low-pass filter to equalized short term fading as well as an hysteresis margin to avoid oscillation of the station between two APs. The authors clearly show how the mobile's velocity affects the handover delay finally yielding to requirements regarding the minimal overlap of adjacent radio cells in order to enable a zero-delay handover. Nevertheless, the work presented in [10]-[14] has two shortcomings: The used attenuation model only accounts for log-normal path loss but not for short term fading. Also, the presented analysis models the low-path filter as a timecontineous process which does not correspond to the discrete sampling of the radio channel used as an input for the filtering process.

Extending the findings of this previous work, this paper's novelties are:

- accounting for a casual, discrete non-recursive low-pass filter applicable to existing implementations;

- the utilization of real channel traces for the performance evaluation; as well as

- the consideration of the effects of having existing radio front-ends on obtaining signal strength samples.

Hereby, this paper presents for the first time a practical verification of the theoretical findings in [12] and [14].

The remainder of the paper is structured as follows: Section II introduces the system model in accordance to [12]. Afterwards, Section III focuses on how to derive the minimal requirements regarding the overlap of adjacent radio cells in order to guarantee a zero delay handover. Therein, we firstly 
present a pure analytical solution assuming an ideal AWGN, log-normal channel model (Section III-A). Secondly, we describe how we use a empirical channel traces in a simulation to obtain equivalent overlap requirements (Section III-B). Finally, Section IV exemplarily compares the results from the analysis and simulation.

\section{SySTEM MODEL}

In accordance to [12], we consider two access points $A P_{1}$ and $A P_{2}$ separated by distance $D$ (c.f. Figure 1). Both APs regularly announce at a frequency $f$ their existence by a pilot tone or frame which also enables acquiring samples of the received signal strength indicator (RSSI) $\mu_{1}(d)$ and $\mu_{2}(d)$ from either AP giving the distance $d$ between mobile to the corresponding AP. The mobile travels in a straight line at velocity $v$ from one AP to the other and may retrieve samples of the received power from either AP without being able to successfully decode the received information as documented in [18]. The mobile exchanges user data with the AP having the larger signal. Hence, a handover from $A P_{1}$ to $A P_{2}$ occurs if $\bar{\mu}_{2}(d) \geq \bar{\mu}_{1}(d)+h$ where $h$ is a hysteresis margin avoiding predominant oscillation between the APs and $\bar{\mu}(\cdot)$ denotes to a received signal after applying a non-casual, discrete lowpass filter having $N$ delay elements (c.f. Fig. 2). Such filtering avoids false handovers due to short term fading. In order to enable a zero-delay handover, the later has to occur while the mobile is within the coverage are of both APs.

For the analytical part, we assume for $\mu(\cdot)$ a log-normal path loss model (ideal AWGN channel) whereas for the simulation, we use a empirical channel model based on channel traces as measured between a high-speed train moving at several hundred $\mathrm{km} / \mathrm{h}$ and a trackside AP operating in the $3.5 \mathrm{GHz}$ band. Details for either of the two channel models are given in the following sections. Also, it should be noted that this system model only focuses on the handover decision phase and does not account for handover delay associated by the network discovery phase nor for any required signal between mobile and AP a prior (association phase) which may be reduced to zero as shown in [9].

\section{DERIVATION OF REQUIRED OVERLAP FOR ZERO-DELAY HANDOVER}

\section{A. Analytical Solution}

1) Channel Modelling: The signal power $\mu$ in $[\mathrm{dB}]$ received by the mobile at distance $d$ is given by

$$
\mu(d)=K_{1}-K_{2} \log (d)
$$

where $K_{1}$ represents the transmission and reception antenna gain, $K_{2}$ the environment-specific attenuation of the channel, and $d$ the distance of the mobile to the AP emitting $\mu$ [19].

2) Low-Pass Filtering: As samples of the received signal strength can only be obtained for each transmission of a beacon frame or pilot, we describe this behavior by a sequence

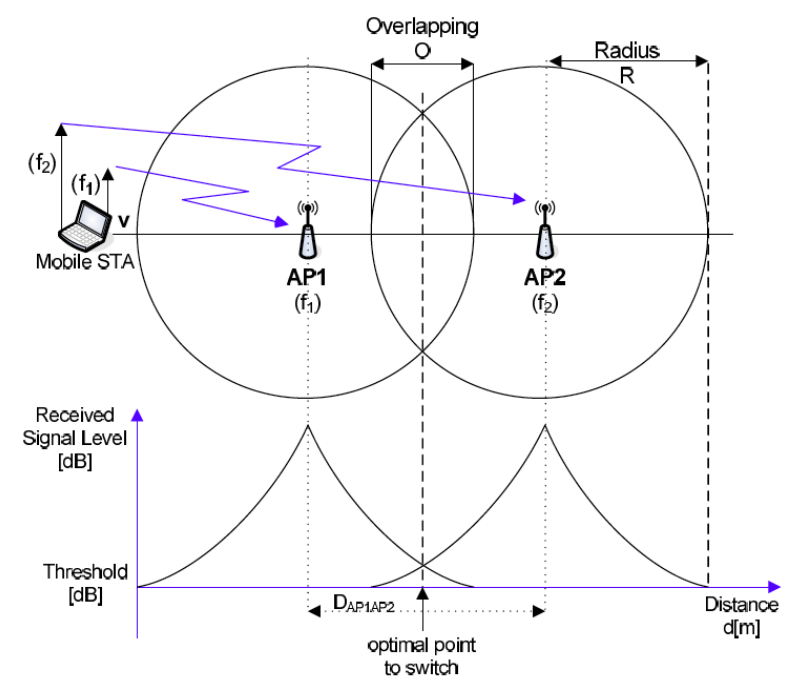

Fig. 1. Handover Scenario

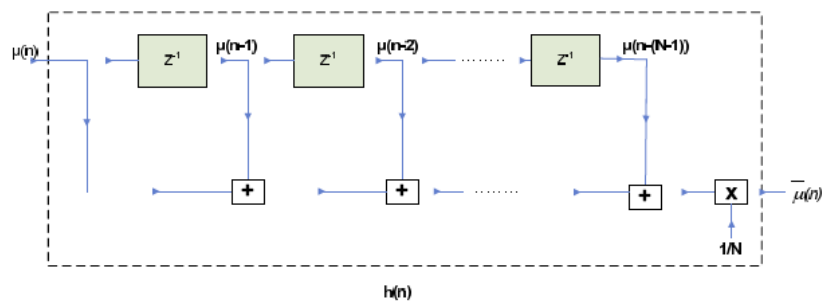

Fig. 2. Low Pass Filtering

of delta impulses $\delta_{\Delta n}(n)=\sum_{-\infty}^{\infty} \delta(n-k \cdot \Delta n)$

$$
\begin{aligned}
\mu[n] \quad=\mu(d) \cdot \delta_{\Delta d}(d) \\
\stackrel{\Delta}{\underline{d}>0} \sum_{k=0}^{\infty} \mu\left(k \cdot \frac{v}{f}\right) \delta(n)
\end{aligned}
$$

The time required to propagate receive power samples from the input of the filter $(\mu[\cdot])$ to its output $(\bar{\mu}[\cdot])$ is given by the group delay $\tau_{g}(\cdot)$

$$
\begin{aligned}
\tau_{g}(\Omega) & =-\frac{\partial \varphi(\Omega)}{\partial \Omega} \\
& =\frac{N-1}{2} \stackrel{N \gg 1}{\approx} \frac{N}{2}
\end{aligned}
$$

where $\varphi(\Omega)=(1-N) \Omega / 2$ is the phase shift of the transfer function of the given filter averaging the last $N$ radio signal strength values [20]. Hence, for a sample frequency $f$, the handover decision employing a low-pass filter $\sigma_{\mathrm{LPF}}$ is delayed by

$$
\begin{aligned}
\sigma_{\mathrm{LPF}} & =\frac{1}{f} \cdot \tau_{g}(\Omega) \\
& =\frac{N-1}{2 f} \approx 1 \frac{N}{2 f}
\end{aligned}
$$


3) Hysteresis Margin: The optimal handover decision involving a hysteresis margin should occur when obtaining the $n_{\text {opt }}$ th sample of the received power such that

$$
n_{\text {opt }}=n \quad: \quad \mu_{2}[n]-\mu_{1}[n] \geq h
$$

which simplifies using Eqs. (3) and (1) to

$$
\begin{aligned}
h & \leq \mu\left[\left\lceil\frac{D f}{v}\right\rceil-n_{\mathrm{opt}}\right]-\mu\left[n_{\mathrm{opt}}\right] \\
& =K_{2} \log \left(\frac{n_{\mathrm{opt}}}{\left\lceil\frac{D f}{v}\right]-n_{\mathrm{opt}}}\right)
\end{aligned}
$$

yielding to

$$
\left\lceil\frac{D f}{v}\right\rceil \frac{10^{\frac{h}{K_{2}}}}{1+10^{\frac{h}{K_{2}}}}=n_{\mathrm{opt}}
$$

assuming that the decision occurs as early as possible, i.e., for the smallest possible $n_{\mathrm{opt}}$. This corresponds to the distance of the mobile to the originating AP given by $n_{\mathrm{opt}} \cdot v / f$ at which the mobile has to be within the overlap of adjacent cells in order to enable a zero handover delay. According to [12], we assume a neglectfully small overlap of adjacent cells requiring the handover to occur in the middle of the two APs at $D / 2$. Passing the latter point without triggering a handover results in a handover delay given by

$$
\begin{aligned}
\sigma_{\mathrm{HM}} & =\frac{\frac{v \cdot n_{\mathrm{opt}}}{f}-\frac{D}{2}}{v} \\
& =\frac{1}{f} \cdot\left\lceil\frac{D f}{v}\right\rceil \frac{10^{\frac{h}{K_{2}}}}{1+10^{\frac{h}{K_{2}}}}-\frac{D}{2 v}
\end{aligned}
$$

which simplifies for $v / f D \cdot\lceil D f / v\rceil \approx 1$ to

$$
\sigma_{\mathrm{HM}} \approx \frac{D}{2 v} \cdot \frac{-1+10^{\frac{h}{K_{2}}}}{1+10^{\frac{h}{K_{2}}}} \leq \frac{D}{2 v}
$$

4) Overlap Requirements for Zero Delay Handover: The total handover delay $\sigma_{\text {total }}=\sigma_{\mathrm{LPF}}+\sigma_{\mathrm{HM}}$ describes the interim between the mobile passing $D / 2$ and the point at which the handover from the old to the new AP is triggered. The latter has to be within the overlapping region to avoid loss of connectivity, hence:

$$
\frac{O}{2} \geq v \cdot \sigma_{\text {total }}=v\left(\sigma_{\mathrm{LPF}}+\sigma_{\mathrm{HM}}\right)
$$

Defining the overlapping ratio $p$ of adjacent cells as

$$
p=\frac{O}{2 \cdot R}
$$

where $R$ is the radius of each AP's coverage area, we can use the relation

$$
2 \cdot R=D+O
$$

in combination with Equations (7), (13), (15) to obtain the minimal required overlap ratio $p_{\min }$ for zero delay handover

$$
p_{\min }=\frac{2 v\left(\frac{N-1}{2 f}+\frac{1}{f}\left\lceil\frac{D f}{v}\right\rceil \frac{10^{\frac{h}{K_{2}}}}{1+10^{\frac{h}{K_{2}}}}-\frac{D}{2 v}\right)}{D+2 v\left(\frac{N-1}{2 f}+\frac{1}{f}\left\lceil\frac{D f}{v}\right\rceil \frac{10^{\frac{h}{K_{2}}}}{1+10^{\frac{h}{K_{2}}}}-\frac{D}{2 v}\right)}
$$

which simplifies for $N \gg 1$ and $v / f D \cdot\lceil D f / v\rceil \approx 1$ to

$$
p_{\min } \approx \frac{\frac{N v}{f}+D \cdot \frac{-1+10^{\frac{h}{K_{2}}}}{1+10^{\frac{h}{K_{2}}}}}{D+\frac{N v}{f}+D \cdot \frac{-1+10^{\frac{h}{K_{2}}}}{1+10^{\frac{h}{K_{2}}}}}
$$

\section{B. Simulation Employing Empirical Channel Model}

1) Empirical Channel Model: The attenuation pattern imposed on the received signal at a given distance is varied according to channel traces. The latter are obtained from the Transrapid high speed (bullet) train passing access points operating at $3.5 \mathrm{GHz}$ and being positioned along the track. For proprietary reasons, Fig 3(a) shows only the moving average of obtained RSSI samples. The magnification in Fig 3(b) reveals that the received signal is actually experiences very severe, short-term fading. In order to allow a comparison with the previously presented analysis, we use the characterization of the receive antenna to convert RSSI samples in $\mathrm{dB}$ values. Hereby, we employ the 10th-degree approximation of the empirical reception characteristics as illustrated in Fig 3(c).

2) Simulation Set-Up: Two access points are positioned $D$ apart and emit every $1 / f$ seconds a frame allowing the mobile to obtain a sample the receive signal strength. If the mobile cannot obtain a sample within $1 / f+\epsilon$ seconds, a value of $-120 \mathrm{~dB}$ is assumed which is well below the receiver sensitivity. The mobile travels at a given, constant velocity $v$ directly form one AP to the other. Only packets received from the AP whose low-pass-fltered receive signal $\bar{\mu}$ is by $h \mathrm{~dB}$ larger than the other's are decoded which allows a quantification of the experienced handover delay in accordance to the "access point transition time metric" defined by the IEEE 802.11 draft recommended practice on wireless performance prediction as the time between the last successful reception of a (user) data frame via the originating $A P$ and the first successful reception via the destination AP [21]. Accordingly, the smallest possible (measurable) handover delay depends on the inter-packet arrival time whose expected mean in this particular set-up should not be below $1 / f .1$

The distance between APs $D$ is increased from a small value (resulting in a large overlap of the APs' coverage) until the expected mean of the access point transition time starts to exceed $1 / f$ with a statistic significance. At this point, the resulting overlap is the one required for a zero delay handover (within the limits of the measurement granularity of $1 / f$ ). To determine the overlap, we herein define the radius $R$ of the coverage area to be the distance, where the expected mean of the received radio signal falls below the radio receiver's reception sensitivity.

\section{COMPARISON OF ANALYSIS AND EMPIRICAL RESUlTS}

Figure 4 shows the minimal required overlap derived from the analysis (using an ideal AWGN channel) and the simulation (using empirical channel traces) for parameters given in

\footnotetext{
${ }^{1}$ Even though we derive the metric characterizing the handover delay from the IEEE 802.11 context, system characteristics and assumptions do not limit the results presented herein to this specific technology as such.
} 


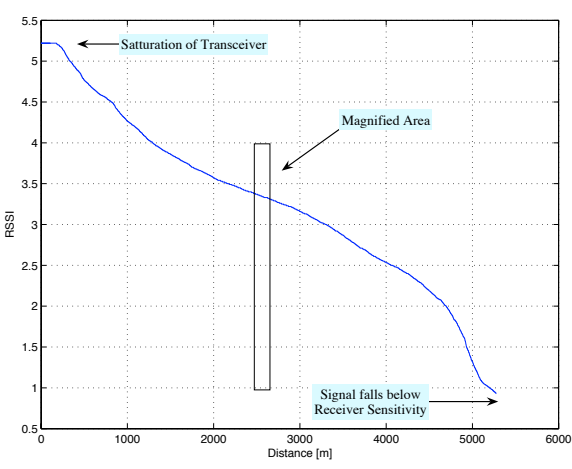

(a) Moving Average of RSSI Samples

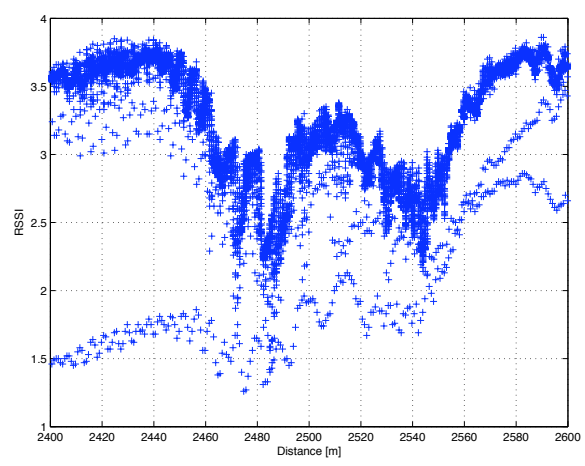

(b) Magnification showing actual RSSI samples

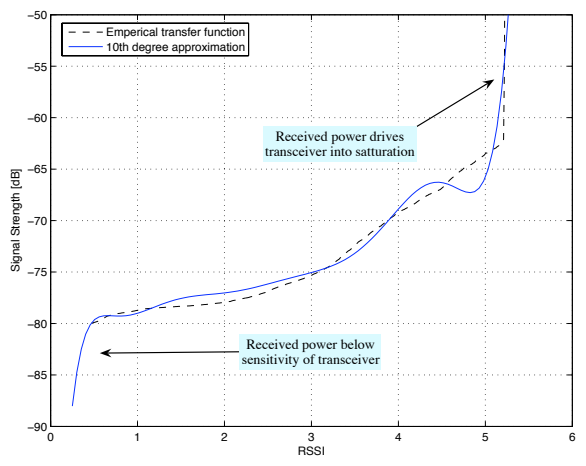

(c) Receive Characteristic of Transceiver

Fig. 3. Empirical Radio Signal Strength over Distance

TABLE II

PARAMETERIZATION OF ANALYSiS AND SimUlation

\begin{tabular}{|c||c|c|}
\hline Parameter & $\begin{array}{c}\text { Value applicable } \\
\text { for presented results }\end{array}$ & $\begin{array}{c}\text { Analyzed parameter } \\
\text { range }\end{array}$ \\
\hline Hysteresis Margin $h$ & $3 \mathrm{~dB}$ & $1-5 \mathrm{~dB}$ \\
Filtered Samples $N$ & 500 & $10-6000$ \\
Sampling frequency $f$ & $1 \mathrm{kHz}$ & dito \\
\hline
\end{tabular}

Table II. ${ }^{2}$ Results differ by less than $1 \%$; for large velocities, analytical results even fall within the $95 \%$ confidence interval of simulation based overlap requirements. Nevertheless, we observed the general trend that overlap requirements obtained from the simulation using real channel traces are slightly below those gained from the analysis which can be explained by the behavior of the underlying channel model. For both results, larger velocities require a larger overlap enabling a zero delay handover. The slight difference in the analytical and empercal results can be explained as follows:

The trace-based channel model does not allow the reception of packets once the mobile leaves the coverage area of the AP. Hence, a sample of the RSSI value cannot be retrieved within $1 / f+\epsilon$ and we feed a value of $-120 \mathrm{~dB}$ in the low pass filter. This value is actually well below the receivers sensitivity threshold (at approximately $-80 \mathrm{~dB}$ and results that the output signal of the low pass filter decreases rather fast once the mobile leaves the AP's coverage area.

In contrast, the analytical, AWGN channel does not model the behavior resulting from such a receiver sensitivity threshold and hence continuously considers channel samples fading over distance at log-normal scale. Hence, the slope of the lowpass filtered signal is lower at large distances and the handoff condition $\bar{\mu}_{2}(d) \geq \bar{\mu}_{1}(d)+h$ is reached at a later time. This results a slightly larger overlap. Spoken from an operator's deployment point of view, this difference can be ignored as it is practically in the order of a few meters.

\footnotetext{
${ }^{2}$ Without loss of generality, we herein present only the results of one parameter set as results resemble qualitatively.
}

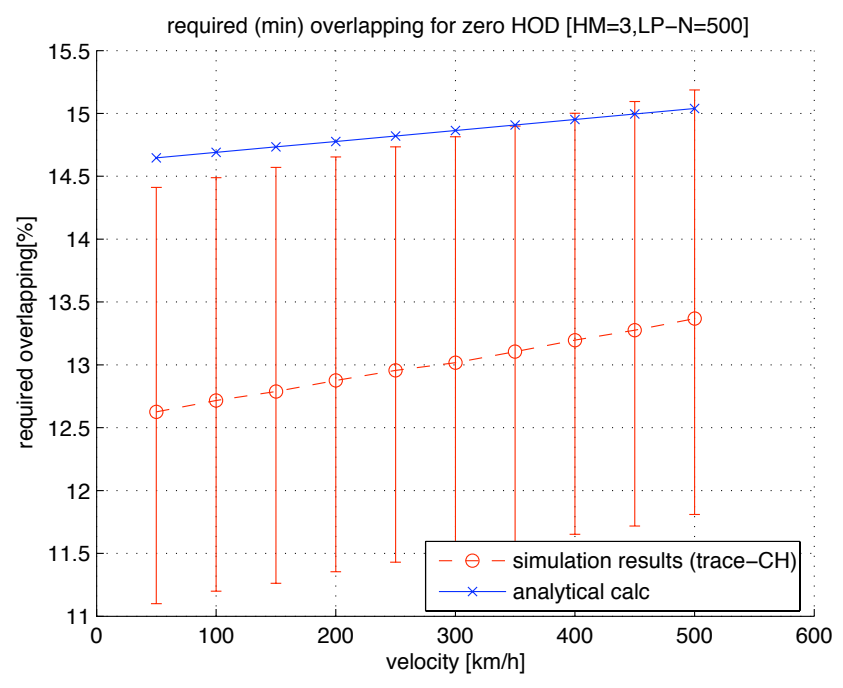

Fig. 4. Minimal required cell overlap for zero-delay handover

\section{SUMmary AND FUtURE WORK}

This paper presented the influence of the mobile's velocity on the overlap requirement of adjacent radio cells if the handover decision is based on radio signal level measurements. Comparing the results from a discrete, analytical model and those of a simulation employing real world channel traces, this paper validated for the first time the mere theoretical findings in [12] assuming a time-continuous acquisition of the channel's attenuation characteristic.

In our future work, we plan to extend the analysis and simulation to a system model where the mobile does not move directly form one AP to the other but instead moves at a random trajectory between multiple APs. Such results will allow to assess overlap requirements for scenarios other than typical vehicular environments for which mobiles are bound to travel along the road / railway track.

\section{REFERENCES}

[1] IEEE 802.11n/D8.0 - Enhancements for Higher Throughput, Draft Amandment to Standard for Information Technology - Telecommunica- 
tions and Information Exchange Between Systems - LAN/MAN Specific Requirements - Part 11: Wireless Medium Access Control (MAC) and physical layer (PHY) specifications, IEEE Std. 802.11n, Rev. D8.0, February 2009.

[2] E. Perahia, "Vht 60 ghz par plus 5c's," IEEE 802.11 VHT SG Very High Throughput Study Group, Denver, CO, USA, doc. 11-08/806, July 2008.

[3] — "Vht60 tutorial," IEEE 802.11 VHT SG Very High Throughput Study Group, Denver, CO, USA, doc. 11-08/814, July 2008.

[4] —-, "Vht below $6 \mathrm{ghz}$ par plus 5c's," IEEE 802.11 VHT SG Very High Throughput Study Group, Denver, CO, USA, doc. 11-08/807, July 2008.

[5] M. de Courville, M. Kamoun, A. Robert, J. Gosteau, R. Fracchia, and S. Gault, "Another resource to exploit: multi-user diversity," IEEE 802.11 VHT SG Very High Throughput Study Group, San Fransico, CA, USA, doc. 11-07/2187, July 2007.

[6] J. Gross, M. Emmelmann, O. Punal, and A. Wolisz, "Dynamic singleuser ofdm adaptation for IEEE 802.11 systems," in Proc. of ACM/IEEE International Symposium on Modeling, Analysis and Simulation of Wireless and Mobile Systems (MSWIM 2007), Chania, Crete Island, October 2007.

[7] —_ "Dynamic multi-user ofdm for 802.11 systems," IEEE 802.11 VHT SG Very High Throughput Study Group, San Francisco, CA, USA, doc. 07/2062, July 2007.

[8] _ , "Performance comparison of dynamic ofdm with 802.11n," IEEE 802.11 VHT (Very High Throughput) SG, Atlanta, GA, USA, doc. 1107/2860, November 12 - 162007.

[9] M. Emmelmann, T. Langgärtner, and M. Sonnemann, "System design and implementation of seamless handover support enabling real-time telemetry applications for highly mobile users," in Proc. of the 6-th ACM International Symposium on Mobility Management and Wireless Access (MobiWac 2008). Association for Computing Machinery (ACM), October 30-31 2008, pp. 1-8.

[10] N. Zhang and J. Holtzman, "Analysis of handoff algorithms using both absolute and relative measurements," Vehicular Technology Conference, 1994 IEEE 44th, vol. 1, pp. 82-86 vol.1, Jun 1994.

[11] N. Zhang and J. Holtzmann, "Analysis of handoff algorithms using both abosute and relative measurements," Vehicular Technology, IEEE Trans. on, vol. 45, no. 11, pp. 174-179, February 1996.

[12] M. Emmelmann, "Influence of velocity on the handover delay associated with a radio-signal-measurement-based handover decision," in Vehicular Technology Conference Fall 2005, Proc. of IEEE 62nd. Dallas, TX, USA: IEEE, September, $26-29$ 2005, extended version.

[13] _ , "Influence of velocity on the handover delay associated with a radio-signal-measurement-based handover decision," TU Berlin, Berlin, Germany, Tech. Rep. TKN-05-003, April 2005.

[14] _ " "Velocity effects on rsm-based handover decision," IEEE 802.11 TGt Wireless Performance Prediction, Plenary Session, Atlanta, GA, USA, doc. 11-05/233, March 2005.

[15] Y.-S. Chen, M.-C. Chuang, and C.-K. Chen, "Deucescan: Deuce-based fast handoff scheme in ieee 802.11 wireless networks," Vehicular Technology, IEEE Transactions on, vol. 57, no. 2, pp. 1126-1141, March 2008.

[16] Y.-S. Chen, C.-K. Chen, and M.-C. Chuang, "Deucescan: Deuce-based fast handoff scheme in ieee 802.11 wireless networks," Vehicular Technology Conference, 2006. VTC-2006 Fall. 2006 IEEE 64th, vol. 1, pp. 1-5, Sept. 2006.

[17] Y. Amir, C. Danilov, M. Hilsdale, R. Musăloiu-Elefteri, and N. Rivera, "Fast handoff for seamless wireless mesh networks," in MobiSys '06: Proceedings of the 4th international conference on Mobile systems, applications and services. New York, NY, USA: ACM, 2006, pp. 83-95.

[18] IEEE 802.11k/D7.0 - Radio Resource Measurement, Draft Amandment to Standard for Information Technology - Telecommunications and Information Exchange Between Systems - LAN/MAN Specific Requirements - Part 11: Wireless Medium Access Control (MAC) and physical layer (PHY) specifications, IEEE Std. 802.11k, Rev. D7.0, January 2007.

[19] A. Aguiar and J. Gross, "Wireless channel models," Technical University of Berlin - Telecommunication Networks Group, Einsteinufer 25, 10587 Berlin Germany, Tech. Rep. TKN-03-007, April 2003. [Online]. Available: http://www.tkn.tu-berlin.de

[20] A. V. Oppenheim, A. S. Willsky, and W. Hamid, Signals and Systems, 2nd ed., ser. Signal Processing. Prentice-Hall, August 1996
[21] IEEE 802.11.2 - Recommended Practice for the Evaluation of 802.11 Wireless Performance, IEEE Std. 802.11, Rev. D1.01, 2008. 